\title{
Kcnk3, Ggta1, and Gpr84 are involved in hyperbaric oxygenation preconditioning protection on cerebral ischemia-reperfusion injury
}

\author{
Chunhui Yang $^{1} \cdot$ Minjun Ding ${ }^{1}$ - Guiqiang Shao ${ }^{2} \cdot$ Shengjie Jia ${ }^{1} \cdot$ Xue Yin ${ }^{1} \cdot$ Yuhui Cui ${ }^{1} \cdot$ Zetian Wang $^{1} \cdot$ Chunrong Wu$^{1}$
}

Received: 8 March 2021 / Accepted: 28 August 2021 / Published online: 30 September 2021

(c) The Author(s) 2021

\begin{abstract}
The present study aimed to explore the potential mechanism of the effect of hyperbaric oxygenation (HBO) preconditioning on cerebral ischemia and reperfusion injury (CIRI). GSE23160 dataset was used to identify differentially expressed genes (DEGs) from striatum between the middle cerebral artery occlusion (MCAO)/reperfusion and sham rats. The gene clusters with continuous increase and decrease were identified by soft clustering analysis in Mfuzz, and functional enrichment analysis of these genes was performed using clusterProfiler package. The intersection set of the genes with significantly altered expression at post-reperfusion 2,8 , and $24 \mathrm{~h}$ were screened in comparison to $0 \mathrm{~h}$ (sham group), and the expression of these genes was detected in the MCAO/reperfusion model and $\mathrm{HBO}$ preconditioning groups by real-time PCR (RT-PCR) and western blotting. A total of 41 upregulated DEGs, and 7 downregulated DEGs were detected, among which the expression of Gpr84 and Ggtal was significantly upregulated at each reperfusion phase as compared to the sham group, while the expression of $K c n k 3$ was significantly downregulated except in the postreperfusion $8 \mathrm{~h}$ in the striatum group. RT-PCR and western blotting analyses showed that the expression of Ggtal, Gpr84, and Kcnk3 genes between the MCAO/reperfusion and sham rats were consistent with the bioinformatics analysis. In addition, the HBO preconditioning reduced the expression of Ggtal and Gpr84 and increased the expression of Kcnk3 in MCAO/reperfusion rats. Kcnk3, Ggtal, and Gpr84 may play a major role in $\mathrm{HBO}-$ mediated protection of the brain against CIRI.
\end{abstract}

Keywords Middle cerebral artery occlusion/reperfusion · Cerebral ischemia and reperfusion injury · Ggtal · Gpr84 · Kcnk3 $\cdot$ Hyperbaric oxygenation preconditioning

\section{Introduction}

Stroke is one of the three major diseases that threaten human health, and the second-leading cause of death globally (Gorelick 2019). A total of 80.1 million stroke cases and 5.5 million deaths were recorded globally in 2016. The age-related incidences of stroke are highest in east Asia, especially China, followed by eastern Europe, ranging from

Communicated by Sreedharan Sajikumar.

Chunhui Yang and Minjun Ding contributed equally to this work.

Chunrong Wu

bamboo_1985@126.com

1 Department of Trauma-Emergency and Critical Care Medicine Center (TECCMC), The Fifth People Hospital of Shanghai Fudan University, Shanghai 200240, China

2 Department of Hyperbaric Oxygen Chamber, Wujing Hospital, Minhang District, Shanghai 200241, China
200 to 354/100,000 person-years (Johnson et al. 2019). In addition to the high fatality rate, stroke also causes a high disability rate, which might bring a great health burden to society (Lancet 2013). Accumulating evidence has indicated that several risk factors, such as hyperlipidemia, hypertension, and smoking, are responsible for the development of stroke (Kumar et al. 2016; Lu et al. 2019). Due to the increased mortality of stroke, the pathogenic mechanisms induced by risk factors need to elucidated urgently for the prevention and treatment of stroke.

Cerebral ischemia and reperfusion (I/R) injury (CIRI) following stroke aggravates brain damage when blood supply is restored (Venkat et al. 2017). Reportedly, inflammatory responses, production of free oxygen free radical, and disruption of brain-blood barrier (BBB) (Lin et al. 2016; Sun et al. 2018) are the primary events for stroke-induced CIRI. Since CIRI is one of the major causes of lethality, the prevention and alleviation of CIRI constitute the key processes for stroke research. Several therapies, such as 
electroacupuncture (Huang et al. 2019) and hyperbaric oxygenation (HBO) preconditioning (Hentia et al. 2018) are currently applied to alleviate CIRI. HBO treatment is an efficient method to ameliorate CIRI via reducing the infarct volume (Lou et al. 2004). Moreover, HBO therapy reduces BBB damage, improves endothelial function and rheology, and decreases local inflammation and edema after CIRI (Hentia et al. 2018; Sun et al. 2018). However, the exact molecular mechanisms of HBO treatment that affect CIRI are only partially known.

The whole transcriptome sequencing is a robust technique for characterizing and quantifying the transcriptome in different stages using bioinformatics analysis (Yang and Kim 2015). In the present study, the transcriptome expression data of GSE23160 dataset that comprised by 24 brain tissue samples from striatum and cortex of post-I/R injured areas at 2, 8, and $24 \mathrm{~h}$ post-reperfusion were downloaded to identify the CIRI-related differentially expressed genes (DEGs) in the rat middle cerebral artery occlusion (MCAO)/reperfusion model as compared to the sham group (Chen et al. 2011) and analyzed their functions. Then, we evaluated the effect of HBO preconditioning on several key DEGs in the rat MCAO/reperfusion model of ischemia by real-time PCR (RT-PCR) and western blotting. The present study aimed to explore the potential treatment targets of CIRI and investigate the molecular mechanisms of $\mathrm{HBO}$ preconditioning to ameliorate the cardiac function after CIRI.

\section{Methods}

\section{Microarray data}

The GSE23160 dataset was downloaded from the Gene Expression Omnibus (GEO, http://www.ncbi.nlm.nih. gov/geo/) and produced on the platform of the Illumina MouseRef-8 v2.0 expression beadchip (GPL6885). The dataset consisted of 24 brain tissue samples from right striatum and cortex (corresponded to infarct area) of post-I/R injured areas, which were extracted at 2,8 , and $24 \mathrm{~h}$ postreperfusion $(n=4)$, respectively, and 4 brain tissue samples each from the striatum and cortex in the sham group.

\section{Data preprocessing}

The original data were read and preprocessed by using Linear Models for Microarray Data (limma, version 3.32.2, https://bioconductor.org/packages/) package in R software (version 3.4.0) (Smyth 2005), in which, the RMA (robust multi-array average) method was applied to convert the probe level data to expression value with backgroundadjusted, quantile normalization, and $\log 2$ transformed values of perfect-match intensities (Irizarry et al. 2003). Then, the probes were annotated. If different probes mapped to the same gene symbol, the mean expression value of those probes was considered as the expression value of the specific gene.

\section{Identification of DEGs and hierarchical cluster analysis}

After preprocessing the data, limma package was utilized for the analysis of the differential expression of genes between cortex and striatum post $-2,-8$, and $-24 \mathrm{~h}$ I/R groups as compared to the corresponding cortex and striatum of sham groups, respectively. The cut-off criterion for screening DEGs was defined as $P$-value $<0.05$ and $\log _{2}$ fold change (FC) $\mid>0.585$. Then, the common DEGs identified between $2 \mathrm{~h} v s$. sham, $8 \mathrm{~h} v s$. sham, and $24 \mathrm{~h} v s$. sham from the cortex and striatum tissue samples were obtained, respectively. In addition, the cluster heatmap was drawn using pheatmap package in $\mathrm{R}$, followed by a complete-linkage clustering method with Pearson's correlation distance.

\section{Soft clustering analysis}

Soft clustering is more accurate than hard clustering with more robustness to noise and less information loss and can assign a gene to several clusters using the fuzzy c-means algorithm with time-course data on the gene expression (Futschik and Carlisle 2005; Kumar 2007). Mfuzz is one of the software packages for soft clustering, in which the membership values of genes in a cluster is generated for reflecting the strength of a gene's association with a cluster (Kumar 2007). Membership value can reveal the similarity of gene expression vectors to each other. To analyze the expression trend of common DEGs with I/R time changes in both cortex and striatum groups, the Mfuzz package (version 2.36.0, https://bioconductor.org/packages/release/bioc/ $\mathrm{html} / \mathrm{Mfuzz} . \mathrm{html}$ ) in $\mathrm{R}$ was used to perform noise-robust soft clustering analysis that could be used for visualizing the time-dependent expression patterns of genes. According to the changes in the gene expression with time, soft clustering could assign genes to several cluster modules, such as continuous increase, continuous decrease, increase first then decrease, and decrease first then increase. The analysis parameters were set as minimum standard deviation $=0.3$; score (membership values) $=0.5$, and c Optimal $=8$. The modules with a continuous increase or continuous decrease were selected for subsequent analysis.

\section{Functional enrichment analysis}

Gene ontology (GO, http://www.geneontology.org) analysis has been widely used to annotate the gene function in biological processes, molecular functions, and cellular component 
categories (Hulsegge et al. 2009). Kyoto Encyclopedia of Genes and Genomes (KEGG, http://www.genome.jp/kegg) is a database containing the information of genes related to metabolic and regulatory pathways (Kanehisa et al. 2017). In the present study, the clusterProfiler package (version 3.4.4, https://bioconductor.org/packages/release/ bioc/html/clusterProfiler.html) in $\mathrm{R}$ was used to conduct the functional analysis for continuous upregulated and downregulated DEGs in both cortex and striatum groups at different I/R times, respectively. The $\mathrm{P}$-value of enriched pathways was calculated by a hypergeometric distribution test. Subsequently, the $P$-value was revised using BH (Benjamini and Hochberg) method, and the adjusted $P$-value $<0.05$ was served as the cut-off criterion for selecting significant enrichment pathways.

\section{Identification of key DEGs in both cortex and striatum groups}

Based on soft clustering, the gene clusters were acquired. Then, overlapped DEGs with continuous upregulation and downregulation in both groups were identified by Venn diagram using Venny 2.1 online tool. Next, we used clusterProfiler package with the same threshold value, as mentioned above, to conduct the GO and KEGG analyses. Finally, several overlapped DEGs with significantly different results were screened further and changed to homologous genes in rats. These overlapped DEGs were identified as key I/Rrelated genes for the subsequent experimental validations followed in this study.

\section{Animals}

A total of 24 male Sprague-Dawley rats (200-260 g, 9-11-weeks-old) were purchased from Shanghai SLAC Laboratory Animal Co., Ltd. The rats were allowed free access to food and water under controlled conditions (room temperature $24 \pm 2{ }^{\circ} \mathrm{C}$, humidity $50 \pm 5 \%$, and lighting with 12-h light/dark cycle). After a week of adaptive feeding, the animals were randomly and equally divided into three groups: $\mathrm{MCAO} /$ reperfusion, sham operation, and $\mathrm{HBO}$ preconditioning $+\mathrm{MCAO} /$ reperfusion. All procedures were approved by the Animal Ethics Committee and performed in accordance with the committee guidelines.

\section{Focal cerebral ischemia-reperfusion model and HBO treatment}

$\mathrm{MCAO} /$ reperfusion model was used to simulate the $\mathrm{I} / \mathrm{R}$ injury in rats by referencing reforming Longa method (Longa et al. 1989). Briefly, rats were anesthetized intraperitoneally using $3 \%$ pentobarbital $(50 \mathrm{mg} / \mathrm{kg}$, Shanghai Rongbai Biological Technology Co., Ltd, China). The left common carotid artery (CCA), external carotid artery (ECA), and internal carotid artery (ICA) were dissociated following a midline neck incision. Then, the proximal part of CCA was clamped, and the ECA was ligatured. A nylon suture (Beijing Cinontech Co., Ltd; 2432A3, Beijing, China) was inserted in the carotid bifurcation of CCA, avoiding the pterygopalatine artery, to enter the internal carotid artery, and it was about $17-20 \mathrm{~mm}$ distal to the middle cerebral artery. After a 2-h ischemic period, reperfusion was performed by withdrawing the suture to restore the blood supply, and rats were euthanized for $24 \mathrm{~h}$ post-reperfusion. The same procedures were conducted in the sham group, except suture insertion was performed on rats.

In the $\mathrm{HBO}$ preconditioning + MCAO/reperfusion group, animals were firstly treated with $\mathrm{HBO}$ in the pressurized oxygen chamber. After flushing pure oxygen until an oxygen pressure of $0.1 \mathrm{MPa}$ was achieved, the rats were subjected to 2.0 atmospheres absolute (ATA) $(0.2 \mathrm{MPa})$ under pure oxygen for $1 \mathrm{~h}$. After $15 \mathrm{~min}$ of constant decompression, the pressure decreased to normal, and the rats were discharged from the chamber. The rats were pretreated with this method once a day for 7 consecutive days. Next, after $3 \mathrm{~h}$ of the last $\mathrm{HBO}$ preconditioning, the rats were utilized to establish $\mathrm{MCAO/reperfusion} \mathrm{models.}$

\section{RT-PCR analysis}

Approximately, 50-100 mg brain (right motor cortex region) samples were obtained from each rat and homogenized. Total RNA was extracted using RNAiso Plus kit (Cat. 9109, TaKaRa, Dalian, China), and reverse transcribed into complementary DNA (cDNA). Then, the quantitative real-time PCR reactions were conducted using Power SYBR Green PCR Master (4,367,659, Applied Biosystems Inc., CA, USA). The reaction conditions were as follows: $95^{\circ} \mathrm{C}$ for $3 \mathrm{~min}, 95^{\circ} \mathrm{C}$ for $10 \mathrm{~s}$, and 40 cycles at $60{ }^{\circ} \mathrm{C}$ for $30 \mathrm{~s}$. The primers of the detected genes are listed in Table 1; GAPDH was used as the reference gene. The relative expression of the genes was calculated by the $2^{-\Delta \Delta \mathrm{Ct}}$ method. All reactions were repeated three times.

Table 1 The primer sequence for each validated gene

\begin{tabular}{ll}
\hline Primer name & Primer sequence $\left(5^{\prime}-3^{\prime}\right)$ \\
\hline GAPDH-rF & AGACAGCCGCATCTTCTTGT \\
GAPDH-rR & CTTGCCGTGGGTAGAGTCAT \\
Ggta1p-rF & ACCGATTCTGCTGAAGACCT \\
Ggta1p-rR & CAAACAGCAGAGCAACCGAG \\
Gpr84-rF & TTCGGACTCCTCCTCTTTACT \\
Gpr84-rR & ACAACTGGCACCAAGACATAA \\
Kcnk3-rF & GCTCCTTCTACTTCGCCATCA \\
Kcnk3-rR & TGACTAGTGTGAGCGGGATG \\
\hline
\end{tabular}




\section{Western blotting}

The protein levels of key genes in brain tissues samples were analyzed by western blotting. The tissue protein extracts were subjected to SDS-PAGE and electrophoresed proteins were then transferred onto PVDF membranes. Antibodies for Gpr84 and Kcnk3 were added to the membranes and the membranes were incubated overnight at $4{ }^{\circ} \mathrm{C}$. The membranes were then washed 3 times, secondary antibodies (anti-rabbit: 1:10,000 and anti-mouse: 1: 5000; Jackson, American) were added, and samples were incubated for $2 \mathrm{~h}$ at room temperature. A western blot imager was used to observe the protein bands after the blots were further washed and developed using an ECL substrate (Beyotime, Nanjing, China).

\section{Statistical analysis}

The expression values were presented as means \pm standard error of mean (SEM). The statistical significance among different groups was analyzed by one-way analysis of variance (ANOVA) with Newman-Keuls multiple comparison posttest, and $P<0.05$ was considered statistically significant. All analyses were conducted using SPSS 22.0 software (IBM Corporation, Armonk, NY, USA), and GraphPad Prism 5 (GraphPad Software, Inc., La Jolla, CA, USA) was utilized to construct graphs.

\section{Results}

\section{Identification of DEGs and hierarchical cluster analysis}

The GSE23160 dataset consists of 26,534 probes corresponding to 18,120 genes. The DEGs identified in the cortex and striatum groups at different reperfusion times are listed in Table 2. A total of 673, 910, and 1414 DEGs were identified in the cortex, and 590, 1062, and 1596 DEGs were identified in the striatum at 2,8 , and $24 \mathrm{~h}$ post-reperfusion, respectively. With the extension of reperfusion time, the number of DEGs increased gradually (Table 2). Compared to the cortex-sham group, a total of 2240 DEGs were obtained in the cortex I/R group, whereas compared to the striatum sham group, a total of 2595 DEGs were obtained in the striatum I/R group. Hierarchical cluster results showed that the DEGs in the same group preferred to cluster together, but the expression differed at 2,8 , and $24 \mathrm{~h}$ post-reperfusion. Thus, these DEGs could distinguish the samples in different groups (Fig. 1).

\section{DEGs with a continuous rising and declining trends were identified}

Based on the time series method in Mfuzz, a total of 8 gene clusters were obtained for the cortex I/R and striatum I/R groups, respectively. With the extension of reperfusion times, different clusters of gene expression changes were identified. In the cortex I/R group, the gene expression values in clusters 2,4 , and 7 showed a rising trend, while those in clusters 5 and 6 showed a downward trend; the gene expression changes in the other clusters were disordered (Fig. 2). In the striatum I/R group, the gene expression values in clusters 4,6 , and 8 showed a rising trend; conversely, clusters 2 and 7 showed a downward trend. In addition, the gene expression values in cluster 1 increased first and then decreased, whereas clusters 3 and 5 decreased first and then increased (Fig. 3). Moreover, in the cortex I/R group, 246 upregulated DEGs were identified in clusters 2,4 , and 7 with a continuous rising trend, and 134 downregulated DEGs in clusters 5 and 6 with a continuous declining trend. In the striatum I/R group, a total of 274 upregulated DEGs were found in clusters 4,6 , and 8 with a continuously rising trend, while 137 downregulated DEGs were found in clusters 2 and 7 with a continuous downward trend (Table 3 ). Thus, we focused on genes with distinct trends.

\section{Functional enrichment analysis}

The results of GO functional enrichment analysis showed that continuous upregulated DEGs in the cortex group were significantly related to leukocyte migration and response to molecule of bacterial origin, while the continuous downregulated DEGs in cortex group were significantly enriched in the inorganic cation transmembrane transport (Kcnk3). In the striatum group, the continuously upregulated DEGs were closely associated with regulation of inflammatory response ( $\mathrm{C} 3$ and $\mathrm{Ctss}$ ) angiogenesis (Ecm1 and $\mathrm{Hspb} 6)$ and response to wounding (Lcp1 and Pros1), but the downregulated DEGs were not significantly enriched in any GO
Table 2 The number of differentially expressed genes identified in each group at different ischemia/reperfusion times

\begin{tabular}{llllllll}
\hline & Cortex & \multicolumn{7}{l}{ Striatum } \\
\cline { 2 - 3 } & $2 \mathrm{~h}$ & $8 \mathrm{~h}$ & $24 \mathrm{~h}$ & & $2 \mathrm{~h}$ & $8 \mathrm{~h}$ & $24 \mathrm{~h}$ \\
\hline Up & 392 & 509 & 815 & 325 & 538 & 899 \\
Down & 281 & 401 & 599 & & 265 & 524 & 697 \\
all & 673 & 910 & 1414 & & 590 & 1062 & 1596 \\
\hline
\end{tabular}




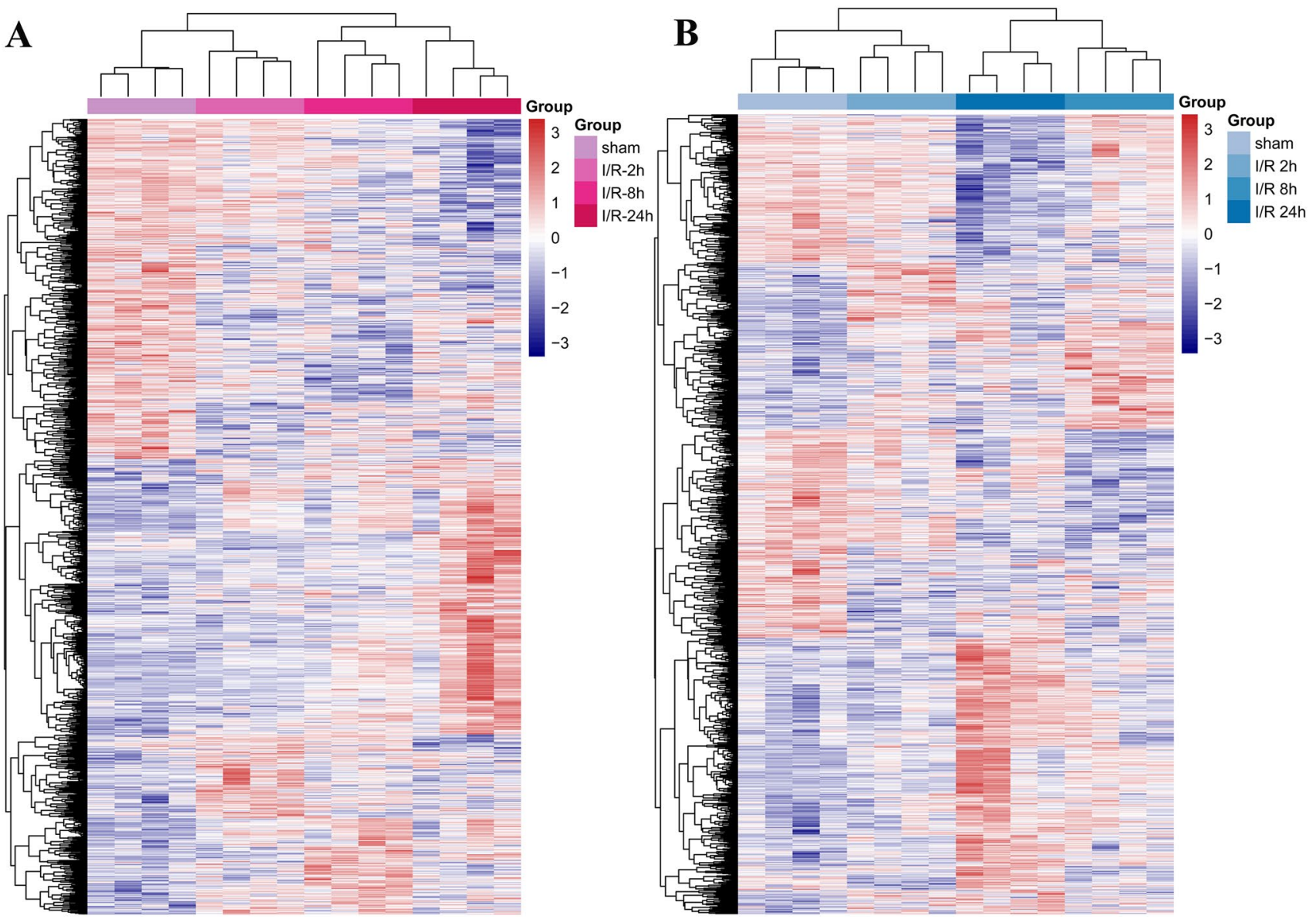

Fig. 1 Hierarchical cluster map of DEGs identified in the cortex (A) and striatum (B) groups. The horizontal axis denotes samples, and the right vertical axis shows clusters of DEGs. The smoothly changed color from blue to red encodes expression values from low to high

terms (Table 4). For KEGG pathways analysis, the continuous upregulated DEGs in the cortex group were primarily involved in the "TNF signaling pathway (Ccl2, Ccl5, Il6, and Mmp9), whereas the continuously downregulated DEGs in the cortex group were strongly related to cAMP signaling pathway and calcium signaling pathway. In the striatum group, the continuously upregulated DEGs were only enriched in two pathways, including ECM-receptor interaction and proteoglycans in cancer; however, no pathways significantly associated with the downregulated DEGs were detected (Table 5).

\section{Identification of key DEGs}

To explore the common change in genes with continuous rise and fall in both cortex and striatum groups, a Venn diagram analysis was used. A total of 41 overlapped upregulated DEGs and 7 overlapped downregulated DEGs were found (Fig. 4). The expression of 5/41 DEGs (Timp1, Gpr84, Ggtal, 4930486L24Rik, and Osmr) was predicted to have a significant difference at each reperfusion time point in the cortex and striatum groups as compared to the sham group. Moreover, the expression trend of Ggtal and Gpr84 was significantly lower P-value at each reperfusion time point in the cortex and striatum groups as compared to the sham group (Fig. 5A and B). However, none of the downregulated DEGs exhibited significant difference at each reperfusion time in cortex while comparing the striatum to the sham group, and the expression of Kcnk3 was downregulated except postreperfusion at $8 \mathrm{~h}$ in the striatum group (Fig. 5C).

\section{Validation by real-time PCR and western blotting}

The expression of three genes (Ggtal, Gpr84, and Kcnk3), was distinct as evaluated by RT-PCR in MCAO/reperfusion, sham operation, and $\mathrm{HBO}$ preconditioning $+\mathrm{MCAO} /$ reperfusion groups (Fig. 6A). The results showed that Ggtal mRNA level was significantly upregulated in $\mathrm{MCAO} /$ reperfusion rats $(P<0.001 v s$. sham $)$, and HBO preconditioning markedly attenuated the Ggtal expression in MCAO/reperfusion rats $(P<0.05)$. Similarly, a significantly increased mRNA expression of Gpr84 was detected 
Fig. 2 Eight clusters identified in the cortex group based on gene expression changes of DEGs in the cortex at post-reperfusion $0,2,8$, and $24 \mathrm{~h}$ by soft cluster analysis. Yellow or green colored curves correspond to genes with low membership values, while curves in red or purple correspond to genes with high membership values. If genes have a high membership values for a cluster, they are generally similar to each other and contribute significantly to overall expression trends of one cluster. Using this color scheme, clusters with a large core of tightly co-regulated genescan be easily distinguished from week or noisy clusters
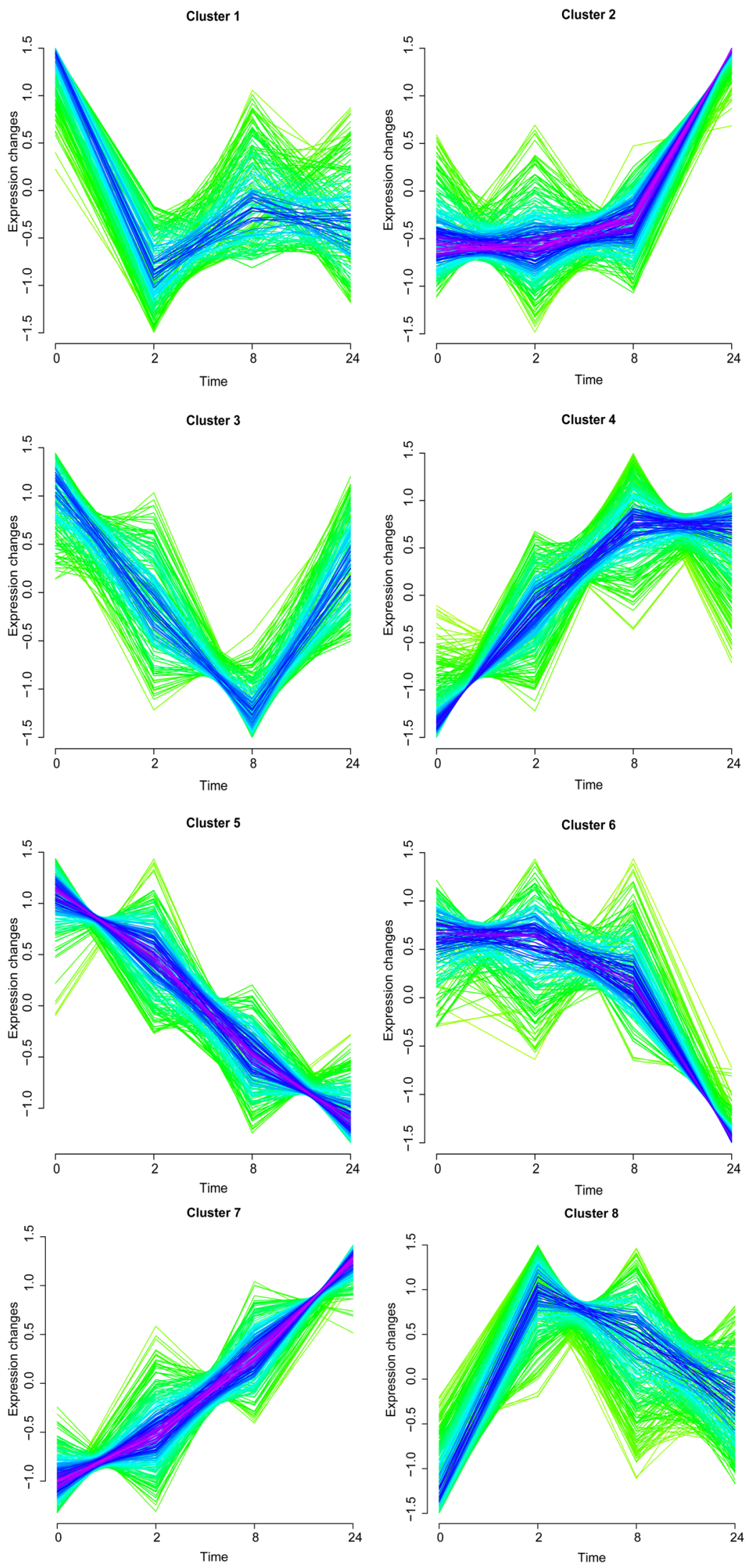
Fig. 3 Eight clusters identified in the striatum group based on gene expression changes of DEGs in the striatum at postreperfusion $0,2,8$, and 24 h by soft cluster analysis. Curves in yellow or green denote genes with small membership values, while curves in red or purple correspond to genes with big membership values
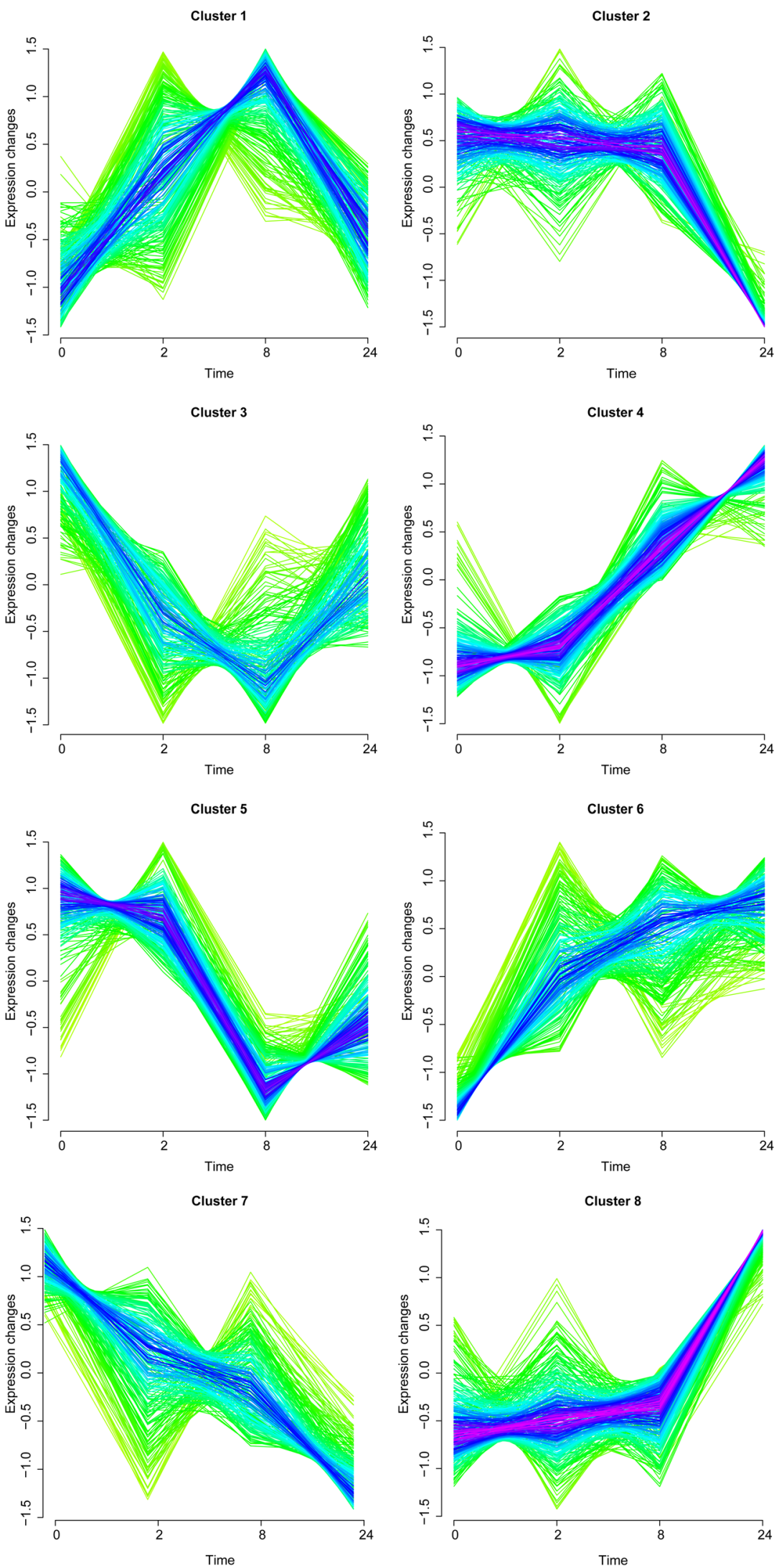
Table 3 The results of soft clustering analysis for differentially expressed genes identified in each group

\begin{tabular}{|c|c|c|c|c|c|c|}
\hline & \multicolumn{3}{|l|}{ Cortex } & \multicolumn{3}{|l|}{ Striatum } \\
\hline & Clusters & Gene counts & Total & Clusters & Clusters & Tota \\
\hline \multirow[t]{3}{*}{ Up } & Cluster 2 & 93 & \multirow[t]{3}{*}{246} & Cluster 4 & 106 & \multirow[t]{3}{*}{274} \\
\hline & Cluster 4 & 49 & & Cluster 6 & 42 & \\
\hline & Cluster 7 & 104 & & Cluster 8 & 126 & \\
\hline \multirow[t]{2}{*}{ Down } & Cluster 5 & 75 & \multirow[t]{2}{*}{134} & Cluster 2 & 98 & \multirow[t]{2}{*}{137} \\
\hline & Cluster 6 & 59 & & Cluster 7 & 39 & \\
\hline
\end{tabular}

Table 4 The top five enriched Go terms for continuous up-regulated and down-regulated genes identified in each group listed by p.adjust value

\begin{tabular}{|c|c|c|c|c|c|}
\hline Category & Term & Description & Count & Gene symbol & p.adjust \\
\hline \multirow[t]{5}{*}{$\begin{array}{l}\text { Up-regulated genes in Cortex } \\
\text { group }\end{array}$} & GO:0,002,237 & $\begin{array}{l}\text { Response to molecule of bacterial } \\
\text { origin }\end{array}$ & 23 & $\begin{array}{l}\text { Ccl12, Cc15, Cd14, Cd84, Cebpb, } \\
\text { Cxcl16, Il1b, Il6, Lbp, Nos3... }\end{array}$ & $1.22 \mathrm{E}-11$ \\
\hline & GO:0,032,496 & Response to lipopolysaccharide & 22 & $\begin{array}{l}\text { Nos3, Nr1h3, Nradd, Pycard, } \\
\text { Slc11a1, Slpi, Ticam2, Tirap, } \\
\text { Tnfrsf1a, Tnfrsf } 22 . .\end{array}$ & $1.95 \mathrm{E}-11$ \\
\hline & GO:0,050,900 & Leukocyte migration & 21 & $\begin{array}{l}\text { Ecm1, Fcer1g, Fcgr3, Hsd3b7, } \\
\text { Icam1, Mmp14, Mmp9, Msn, } \\
\text { Pycard, Tirap... }\end{array}$ & 4.44E-11 \\
\hline & GO:0,001,819 & $\begin{array}{l}\text { Positive regulation of cytokine } \\
\text { production }\end{array}$ & 23 & $\begin{array}{l}\text { Bcl3, C3, Ccl2, Cc15, Cd14, } \\
\text { Clec5a, Fcer1g, Tirap, Tlr1, } \\
\text { Tnfrsf1a... }\end{array}$ & $4.63 \mathrm{E}-11$ \\
\hline & GO:0,002,685 & Regulation of leukocyte migration & 16 & $\begin{array}{l}\text { Anxa1, Ccl12, Cc12, Ecm1, Icam1, } \\
\text { Il1b, Lbp, Mmp14, Mmp9, } \\
\text { Tirap... }\end{array}$ & 4.37E-10 \\
\hline \multirow[t]{5}{*}{$\begin{array}{l}\text { Down-regulated genes in Cortex } \\
\text { group }\end{array}$} & GO:0,007,611 & Learning or memory & 12 & $\begin{array}{l}\text { Drd2, Fgf13, Grin2b, Htr2a, Jph4, } \\
\text { Musk, Nf1, Nog, Pak7, Pde1b... }\end{array}$ & 7.86E-07 \\
\hline & GO:0,007,613 & Memory & 9 & $\begin{array}{l}\text { Cnr1, Cpeb3, Drd2, Fgf13, } \\
\text { Grin2b, Htr2a, Musk, Nog, Pak7 }\end{array}$ & 8.97E-07 \\
\hline & GO:0,050,890 & Cognition & 12 & $\begin{array}{l}\text { Drd2, Fgf13, Grin2b, Htr2a, Jph4, } \\
\text { Musk, Nf1, Nog, Pak7, Pde1b... }\end{array}$ & 8.97E-07 \\
\hline & GO:0,044,708 & Single-organism behavior & 13 & $\begin{array}{l}\text { Alk, Cnr1, Grin2b, Htr2a, Jph4, } \\
\text { Musk, Nf1, Nog, Pak7, Pde1b... }\end{array}$ & $3.11 \mathrm{E}-05$ \\
\hline & GO:0,098,662 & $\begin{array}{l}\text { Inorganic cation transmembrane } \\
\text { transport }\end{array}$ & 13 & $\begin{array}{l}\text { Cacng7, Cacng8, Drd2, Htr2a, } \\
\text { Kcnd2, Kcnh3, Kcnk3, Pcsk9, } \\
\text { Scn4b... }\end{array}$ & $3.30 \mathrm{E}-05$ \\
\hline \multirow[t]{5}{*}{$\begin{array}{l}\text { Up-regulated genes } \\
\text { in Striatum group }\end{array}$} & GO:0,031,589 & Cell-substrate adhesion & 15 & $\begin{array}{l}\text { Jam3, Msln, Myo1g, Notch1, } \\
\text { Otoa, Plau, S100a10, Sdc4, } \\
\text { Spp1, Tnn... }\end{array}$ & 2.69E-05 \\
\hline & GO:0,007,160 & Cell-matrix adhesion & 11 & $\begin{array}{l}\text { Col3a1, Gpm6b, Iqgap1, Jam3, } \\
\text { Msln, Otoa, Plau, S100a10, } \\
\text { Sdc4, Tnn... }\end{array}$ & 0.000111 \\
\hline & GO:0,009,611 & Response to wounding & 16 & $\begin{array}{l}\text { Hspb1, Lcp1, Lox, Map2k1, Plau, } \\
\text { Pros1, S100a9, Sdc4, Slc11a1, } \\
\text { Tnc... }\end{array}$ & 0.000111 \\
\hline & GO:0,050,727 & $\begin{array}{l}\text { Regulation of inflammatory } \\
\text { response }\end{array}$ & 13 & $\begin{array}{l}\text { C3, Casp1, Ctss, Fcgr2b, Mefv, } \\
\text { Myd88, Nr1d2, S100a8, S100a9, } \\
\text { Tlr3... }\end{array}$ & 0.000111 \\
\hline & GO:0,001,525 & Angiogenesis & 16 & $\begin{array}{l}\text { Esm1, Hmox1, Hspb1, Hspb6, } \\
\text { Ihh, Jam3, Lif, Lrg1, Notch1, } \\
\text { Thbs2... }\end{array}$ & 0.000111 \\
\hline
\end{tabular}

Note: $G O$ Gene ontology 
Table 5 The top five enriched pathways for continuous up-regulated and down-regulated genes identified in each group listed by p.adjust value

\begin{tabular}{|c|c|c|c|c|c|}
\hline Category & Term & Description & Count & Gene symbol & p.adjust \\
\hline \multirow[t]{5}{*}{ Up-regulated genes in Cortex group } & mmu04668 & TNF signaling pathway & 12 & $\begin{array}{l}\text { Bcl3, Ccl12, Ccl2, Ccl5, Cebpb, } \\
\text { Icam1, Il1b, Il6, Mlk1, Mmp14, } \\
\text { Mmp9, Tnfrsf1a }\end{array}$ & $1.07 \mathrm{E}-05$ \\
\hline & mmu05144 & Malaria & 8 & $\begin{array}{l}\text { Ccl12, Ccl2, Hba-a1, Hbb-b1, } \\
\text { Icam1, Il1b, Il6, Selp }\end{array}$ & 4.80E-05 \\
\hline & mmu05133 & Pertussis & 9 & $\begin{array}{l}\text { C1qb, C3, C4b, Cd14, I11b, I16, } \\
\text { Pycard, Ticam2, Tirap }\end{array}$ & $9.08 \mathrm{E}-05$ \\
\hline & mmu05152 & Tuberculosis & 13 & $\begin{array}{l}\text { C3, Cd14, Cebpb, Fcer1g, Fcgr1, } \\
\text { Fcgr3, Il1b, I16, Lbp, Lsp1, Tirap, } \\
\text { Tlr1, Tnfrsf1 }\end{array}$ & 0.000101 \\
\hline & mmu05150 & Staphylococcus aureus infection & 7 & $\begin{array}{l}\text { C1qb, C3, C4b, Fcgr1, Fcgr3, } \\
\text { Icam1, Selp }\end{array}$ & 0.000337 \\
\hline \multirow[t]{5}{*}{$\begin{array}{l}\text { Down-regulated genes in Cortex } \\
\text { group }\end{array}$} & mmu04024 & cAMP signaling pathway & 8 & $\begin{array}{l}\text { Atp2b1, Atp2b4, Drd2, Gria3, } \\
\text { Grin2b, Htr1d, Rock2, Sstr2 }\end{array}$ & 0.00716 \\
\hline & $\mathrm{mmu} 05033$ & Nicotine addiction & 4 & Gabrg1, Gria3, Grin2b, Slc17a8 & 0.009442 \\
\hline & mmu04020 & Calcium signaling pathway & 7 & $\begin{array}{l}\text { Atp2b1, Atp2b4, Htr2a, Mylk2, } \\
\text { Pde1b, Plcz1, Ryr1 }\end{array}$ & 0.010304 \\
\hline & mmu05032 & Morphine addiction & 5 & $\begin{array}{l}\text { Gabrg1, Gnb4, Pde10a, Pde1b, } \\
\text { Pde7b }\end{array}$ & 0.012376 \\
\hline & mmu04261 & $\begin{array}{l}\text { Adrenergic signaling in cardiomyo- } \\
\text { cytes }\end{array}$ & 6 & $\begin{array}{l}\text { Atp2b1, Atp2b4, Cacna2d1, } \\
\text { Cacng7, Cacng8, Scn4b }\end{array}$ & 0.012376 \\
\hline \multirow[t]{2}{*}{$\begin{array}{l}\text { Up-regulated genes in Striatum } \\
\text { group }\end{array}$} & mmu04512 & ECM-receptor interaction & 6 & $\begin{array}{l}\text { Col1a1, Sdc4, Spp1, Thbs2, Tnc, } \\
\text { Tnn }\end{array}$ & 0.025936 \\
\hline & mmu05205 & Proteoglycans in cancer & 9 & $\begin{array}{l}\text { Col1a1, Ihh, Iqgap1, Map2k1, Msn, } \\
\text { Plau, Plaur, Rras2, Sdc4 }\end{array}$ & 0.025936 \\
\hline
\end{tabular}

Fig. 4 The Venn diagram of overlapped upregulated genes and shared downregulated genes in both cortex and striatum groups, respectively. A total of 41 overlapped upregulated DEGs and 7 overlapped downregulated DEGs were identified in both groups

\section{Striatum-Up Cortex-Down}

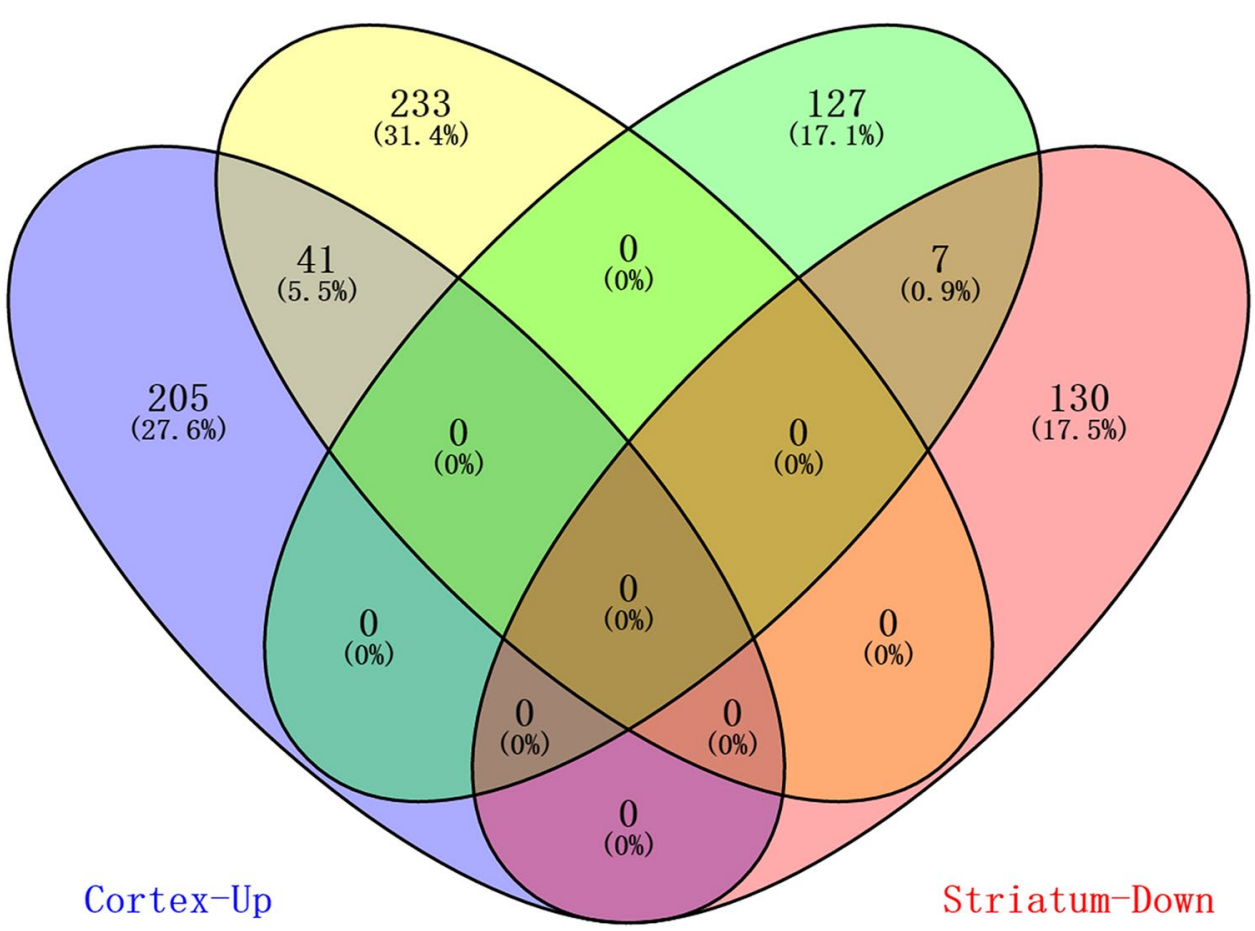



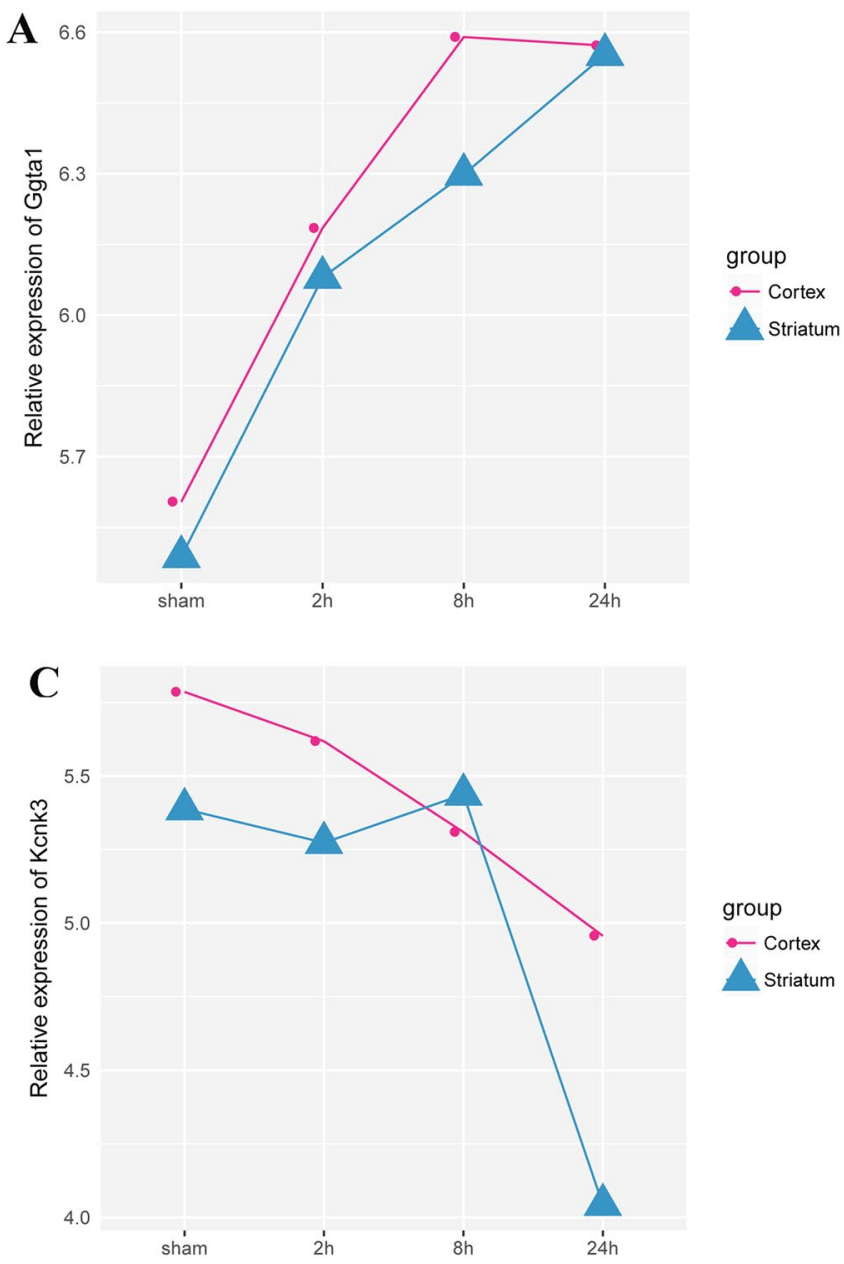

Fig. 5 The expression trends of Ggtal (A), Gpr84 (B), and Kcnk3 (C) at 2, 8, and $24 \mathrm{~h}$ post-reperfusion identified in both cortex and striatum groups. The expression of Ggtal and Gpr84 was upregulated

in $\mathrm{MCAO} /$ reperfusion rats $(P<0.001 v s$. sham $)$, and $\mathrm{HBO}$ preconditioning suppressed the expression of Gpr84 in $\mathrm{MCAO} /$ reperfusion rats significantly $(P<0.01)$. In addition, the mRNA expression of Kcnk3 was significantly downregulated in $\mathrm{MCAO} /$ reperfusion rats $(P<0.001 v s$. sham), and after $\mathrm{HBO}$ preconditioning, the expression was only slightly upregulated in these rats.

In keeping with the RT-PCR analysis, the data of western blotting revealed that the protein level of Gpr84 in $\mathrm{MCAO} /$ reperfusion rats was significantly upregulated in comparison to that in sham controls $(P<0.01$; Fig. 6B); moreover, HBO preconditioning could significantly suppress the expression of Gpr84 protein in MCAO/reperfusion rats $(\mathrm{P}<0.01)$. In contrast, the expression of Kcnk3 protein in $\mathrm{MCAO} /$ reperfusion rats was significantly downregulated $(P<0.01 \mathrm{vs}$. sham $)$, while it was up-regulated after HBO pretreatment $(P<0.01)$.

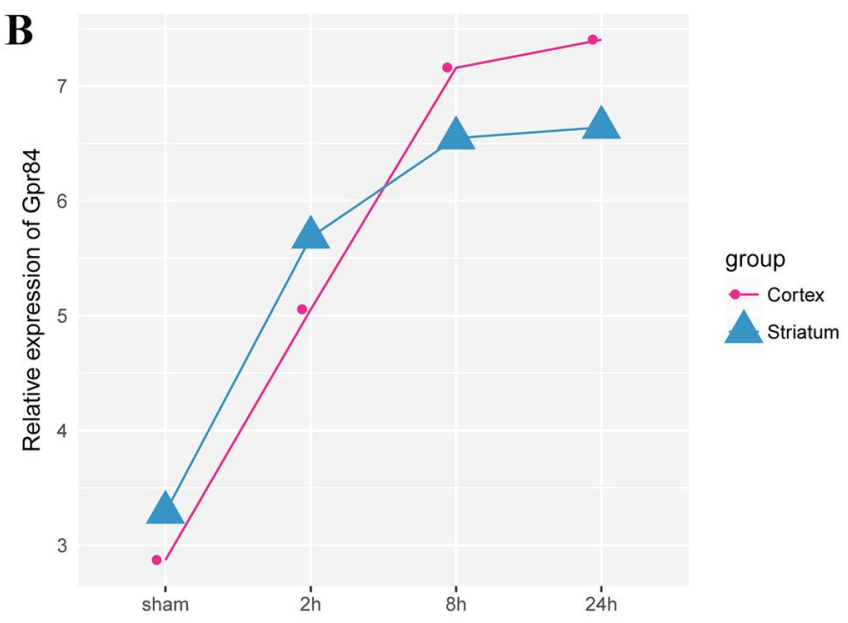

with prolonged post-reperfusion time, while Kcnk3 presented downregulation prolonged post-reperfusion time except for post-reperfusion $8 \mathrm{~h}$ in the striatum group

\section{Discussion}

In the present study, a set of 41 overlapped upregulated DEGs and 7 overlapped downregulated DEGs was found in the cortex and striatum groups, among which the upregulated expression of Gpr84 and Ggtal was significantly different at each reperfusion time points in both groups, while that of downregulated Kcnk3 was distinct. RT-PCR and western blotting analyses showed that the expression of the Ggtal, Gpr84, and Kcnk3 genes between MCAO/ reperfusion and sham rats was consistent with the results of bioinformatics analysis described above. In addition, the expression of these three genes was significantly altered after HBO preconditioning. The current findings revealed new genes that might play critical roles in CIRI, and the protective effect induced by $\mathrm{HBO}$ preconditioning 

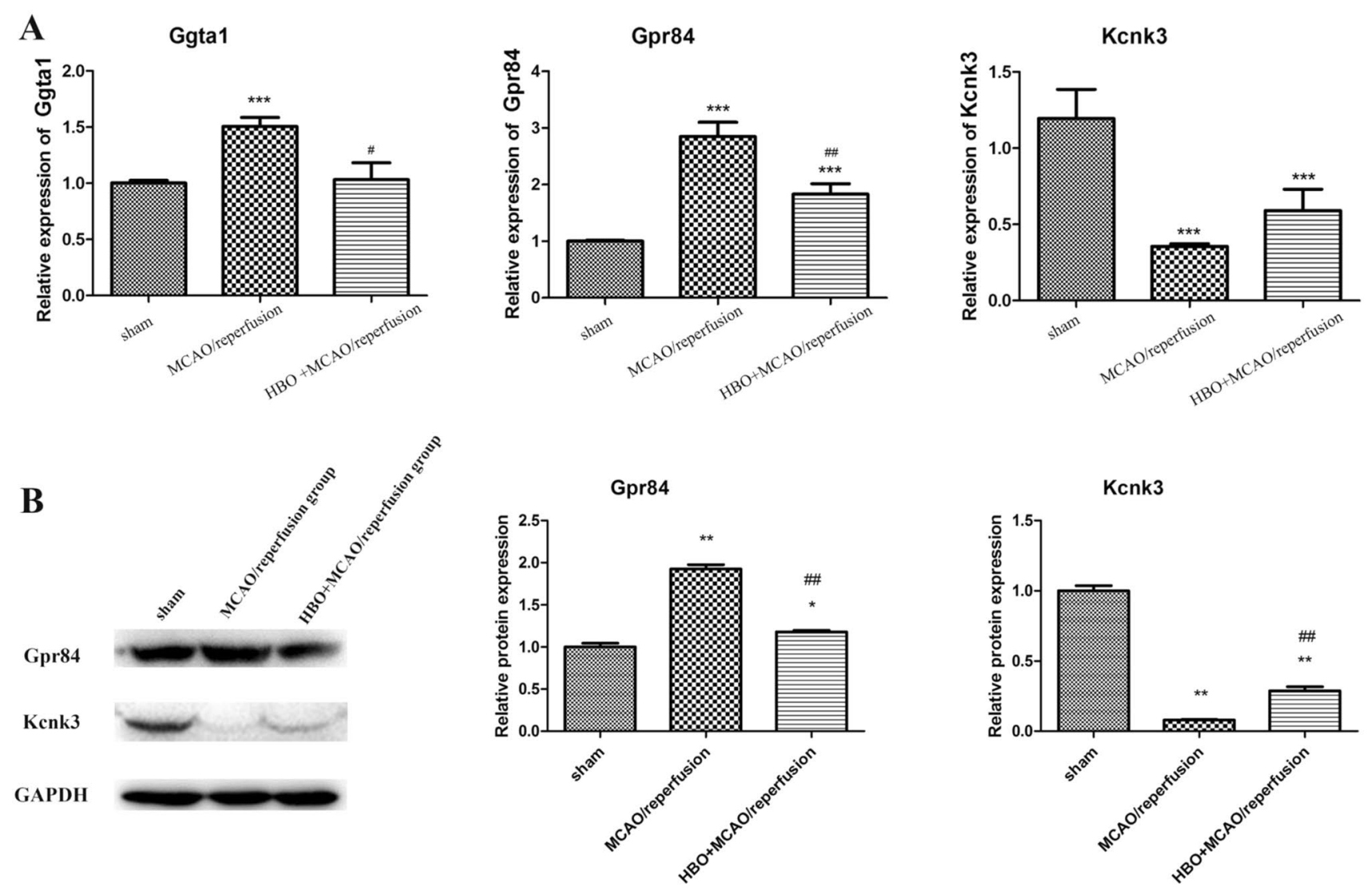

Fig. 6 The relative expression of key genes. The mRNA levels of Ggtal, Gpr84, and Kcnk3 (A) detected by real-time PCR. The protein levels of Gpr84 and Kcnk3 measured by western blotting. MCAO middle cerebral artery occlusion, $H B O$ hyperbaric oxygenation. *, **, and *** represent $P<0.05, P<0.01$, and $P<0.001 \mathrm{vs}$. sham operation group, respectively. \#, \#\#, and \#\#\# represent $P<0.05, P<0.01$, and $P<0.001 v s$. MCAO/reperfusion group, respectively might be associated with DEGs such as Ggtal, Gpr84, and Kcnk3 for ameliorating the subsequent CIRI.

Glycoprotein, alpha-galactosyltransferase 1 pseudogene (Ggtal, also named GLYT2) encodes a terminal glycosyltransferase, which generates a carbohydrate antigen; namely, $\alpha$-gal epitopes (Gal $\alpha 1-3 \mathrm{Gal} \beta 1-4 \mathrm{GlcNAc-R}$ ) (Shaper et al. 1992). Typically, Ggtal is inactivated in human beings, which might lead to remove the $\alpha$-gal epitopes and produce abundant natural anti-Gal antibody in the human (Galili 2015). Conversely, the activated Ggtal may reduce the abundance of natural anti-Gal antibody. anti-Gal antibody is shown to maintain the immunological balance between autoimmunity and infection in humans (Jaison et al. 1993), and Ggtal knockout in mice promotes anti-Gal-mediated increase in immunogenicity (Abdelmotal et al. 2009). In addition, Ggtal is reported to be overexpressed after traumatic brain injury in rats (Samal et al. 2015). In the present study, we detected that the expression of Ggtal was significantly upregulated in the $\mathrm{MCAO} /$ reperfusion group, and the expression was significantly reduced after $\mathrm{HBO}$ preconditioning. Thus, we speculated that the downregulated Ggtal induced by $\mathrm{HBO}$ preconditioning might ameliorate the brain damage in MCAO/reperfusion rats with decreased immunogenicity. However, additional experiments are required to verify this hypothesis.

G-protein-coupled receptor $84(G p r 84)$ is a receptor of medium-chain free fatty acid with a crucial role in fatty acid metabolism that regulates the immune system (Suzuki et al. 2013). Masakatsu et al. reported that the activated $G p r 84$ as a proinflammatory receptor and may not only accumulate the polymorphonuclear leukocytes and macrophages but also mediate lipopolysaccharide-induced IL- 8 and TNF- $\alpha$ production (Suzuki et al. 2013). In addition, Venkataraman et al. demonstrated that in GPR84-knockout $\mathrm{T}$ cells, the expression of antiinflammatory factor IL-4 is increased, which implied that the overexpression of GPR84 mediates the downregulation of IL-4 and in turn, promotes inflammatory response (Venkataraman and Kuo 2005). Inflammation is the main pathogenesis for cerebral ischemia, and a marked inflammatory reaction is evoked by cerebral $\mathrm{I} / \mathrm{R}$ with increased pro-inflammatory factors, such as TNF $\alpha$, IL- $1 \beta$, and IL-6 
(Collino et al. 2006; Junjie et al. 2016). Moreover, the IL-4 knockout after cerebral ischemia might increase brain damage and inflammation (Xiong et al. 2011). In the present study, Gpr84 was found to be significantly upregulated in MCAO/reperfusion rats as compared to sham rats; however, after HBO preconditioning, the expression of Gpr84 was decreased. Thus, we speculated that the HBO preconditioning-induced decrease in Gpr 84 might protect the brain against CIRI by reducing the expressions of inflammation molecules. However, additional experiments are needed to verify this hypothesis.

Potassium channel subfamily $\mathrm{K}$ member 3 (KCNK3, also known as TASK-1) belongs to a member of potassium channel proteins, and the suppression of $K C N K 3$ acts as a leading player in the pathogenesis of pulmonary arterial hypertension pathogenesis with increased proliferation and inflammation (Antigny et al. 2016). Muhammad et al. found that $K C N K 3$ knockout in rat brains might expand the infarct volumes, resulting in a large brain injury, thereby influencing the blood vessel microarchitecture and blood flow rate (Muhammad et al. 2010). Consistent with the current findings, KCNK3 was significantly downregulated in $\mathrm{MCAO} /$ reperfusion rats, and $\mathrm{HBO}$ preconditioning increased the expression of $K C N K 3$ in $\mathrm{MCAO} /$ reperfusion rats slightly. Several studies suggested that $K C N K 3$ is a key form of oxygen-sensitive background $\mathrm{K}+$ channel in rat carotid body glomus cells, and hypoxia can induce the inhibition of TASK-1-like channels (Kim et al. 2009; PJ and KJ 2013). Collectively, we inferred that the upregulation of $K C N K 3$ induced by $\mathrm{HBO}$ preconditioning ameliorates the brain damage in $\mathrm{MCAO} /$ reperfusion rats. However, the underlying mechanism needs to be elucidated further.

In summary, the current study showed that HBO treatment might reduce brain damages and protect the brain against CIRI via altered gene expression alteration of Ggta1, Gpr84, and Kcnk3. Nonetheless, the protective mechanisms and pathways of Ggtal, Gpr84, and Kcnk against CIRI after HBO treatment need further investigation.

Author contributions YCH, YX, JSJ, DMJ, SGQ, WCR conceived and designed the experiments; YCH, YX, JSJ, CYH, WZT, WCR performed the experiments; DMJ, CYH, SGQ, WCR analyzed and interpreted the data; YCH, YX, JSJ, SGQ contributed reagents, materials, analysis tools or data; SGQ, WCR wrote the paper.

\section{Funding None.}

Availability of data and materials The datasets used and/or analysed during the current study are available from the corresponding author on reasonable request.

Code availability Not applicable.

\section{Declarations}

Conflict of interest All authors declare that they have no any conflict of interests.

Ethics approval All procedures were approved by the Animal Ethics Committee of the Fifth People Hospital of Shanghai Fudan University and performed in accordance with the committee guidelines.

Consent to participate Not applicable.

Consent for publication Not applicable.

Open Access This article is licensed under a Creative Commons Attribution 4.0 International License, which permits use, sharing, adaptation, distribution and reproduction in any medium or format, as long as you give appropriate credit to the original author(s) and the source, provide a link to the Creative Commons licence, and indicate if changes were made. The images or other third party material in this article are included in the article's Creative Commons licence, unless indicated otherwise in a credit line to the material. If material is not included in the article's Creative Commons licence and your intended use is not permitted by statutory regulation or exceeds the permitted use, you will need to obtain permission directly from the copyright holder. To view a copy of this licence, visit http://creativecommons.org/licenses/by/4.0/.

\section{References}

Abdelmotal UM, Wigglesworth K, Galili U (2009) Mechanism for increased immunogenicity of vaccines that form in vivo immune complexes with the natural anti-Gal antibody. Vaccine 27:3072

Antigny F, Hautefort A, Meloche J, Belacel-Ouari M, Manoury B, Rucker-Martin C, Péchoux C, Potus F, Nadeau V, Tremblay E, Ruffenach G, Bourgeois A, Dorfmüller P, Breuils-Bonnet S, Fadel E, Ranchoux B, Jourdon P, Girerd B, Montani D, Provencher S, Bonnet S, Simonneau G, Humbert M, Perros F (2016) Potassium channel subfamily $\mathrm{K}$ member 3 (KCNK3) contributes to the development of pulmonary arterial hypertension. Circulation 133:1371-1385. https://doi.org/10.1161/circulationaha.115. 020951

Chen MJ, Wong CH, Peng ZF, Manikandan J, Melendez AJ, Tan TM, Crack PJ, Cheung NS (2011) A global transcriptomic view of the multifaceted role of glutathione peroxidase- 1 in cerebral ischemicreperfusion injury. Free Radic Biol Med 50:736-748. https://doi. org/10.1016/j.freeradbiomed.2010.12.025

Collino M, Aragno M, Mastrocola R, Benetti E, Gallicchio M, Dianzani C, Danni O, Thiemermann C, Fantozzi R (2006) Oxidative stress and inflammatory response evoked by transient cerebral ischemia/ reperfusion: effects of the PPAR-alpha agonist WY14643. Free Radic Biol Med 41:579-589. https://doi.org/10.1016/j.freeradbio med.2006.04.030

Futschik ME, Carlisle B (2005) Noise-robust soft clustering of gene expression time-course data. J Bioinform Comput Biol 3:965-988. https://doi.org/10.1142/s0219720005001375

Galili U (2015) Significance of the evolutionary $\alpha 1,3$ galactosyltransferase (GGTA1) gene inactivation in preventing extinction of apes and old world monkeys. J Mol Evol 80:1-9. https://doi.org/10.1007/s00239-014-9652-x

Gorelick PB (2019) The global burden of stroke: persistent and disabling. Lancet Neurol 18:417-418. https://doi.org/10.1016/s14744422(19)30030-4 
Hentia C, Rizzato A, Camporesi E, Yang Z, Muntean DM, Săndesc D, Bosco G (2018) An overview of protective strategies against ischemia/reperfusion injury: the role of hyperbaric oxygen preconditioning. Brain Behav 8:e00959. https://doi.org/10.1002/ brb3.959

Huang YG, Tao W, Yang SB, Wang JF, Mei ZG, Feng ZT (2019) Autophagy: novel insights into therapeutic target of electroacupuncture against cerebral ischemia/ reperfusion injury. Neural Regen Res 14:954-961. https://doi.org/10.4103/1673-5374. 250569

Hulsegge I, Kommadath A, Smits MA (2009) Globaltest and GOEAST: two different approaches for gene ontology analysis. BMC Proc 3(Suppl 4):S10. https://doi.org/10.1186/1753-6561-3-s4-s10

Irizarry RA, Hobbs B, Collin F, Beazerbarclay YD, Antonellis KJ, Scherf U, Speed TP (2003) Exploration, normalization, and summaries of high density oligonucleotide array probe level data. Biostatistics 4:249-264

Jaison P, Kannan V, Geetha M, Appukuttan P (1993) Epitopes recognized by serum anti- $\alpha$-galactoside antibody are present on brain glycoproteins in man. J Biosci 18:187-193

Johnson C, Nguyen M, Roth G, Nichols E, Alam T, Abate D (2019) Global, regional, and national burden of stroke, 1990-2016: a systematic analysis for the Global Burden of Disease Study 2016. Lancet Neurol 18:439-458. https://doi.org/10.1016/s14744422(19)30034-1

Junjie L, Jiang H, Shao J (2016) The gene expression change of inflammatory factors TNF- $\alpha$, IL- 6 and IL- $1 \beta$ in cerebral ischemia-reperfusion rats. J Kunm Med University 37:31-35

Kanehisa M, Furumichi M, Tanabe M, Sato Y, Morishima K (2017) KEGG: new perspectives on genomes, pathways, diseases and drugs. Nucleic Acids Res 45:D353-d361. https://doi.org/10.1093/ nar/gkw1092

Kim D, Cavanaugh EJ, Kim I, Carroll JL (2009) Heteromeric TASK-1/ TASK- 3 is the major oxygen-sensitive background $\mathrm{K}+$ channel in rat carotid body glomus cells. J Physiol 587:2963-2975. https:// doi.org/10.1113/jphysiol.2009.171181

Kumar L (2007) Mfuzz: a software package for soft clustering of microarray data. Bioinformation 2:5-7. https://doi.org/10.6026/ 97320630002005

Kumar R, Lakhair MA, Memon Z (2016) Ischemic stroke. Profess Med J 23:925-931

Lancet T (2013) Global burden of disease study 2010. Dialyse Aktuell 17:353-353

Lin L, Wang X, Yu Z (2016) Ischemia-reperfusion injury in the brain: mechanisms and potential therapeutic strategies. Biochem Pharmacol (los Angel). https://doi.org/10.4172/2167-0501.1000213

Longa EZ, Weinstein PR, Carlson S, Cummins R (1989) Reversible middle cerebral artery occlusion without craniectomy in rats. Stroke 20:84-91. https://doi.org/10.1161/01.str.20.1.84

Lou M, Eschenfelder CC, Herdegen T, Brecht S, Deuschl G (2004) Therapeutic window for use of hyperbaric oxygenation in focal transient ischemia in rats. Stroke 35:578-583. https://doi.org/10. 1161/01.str.0000111599.77426.a0

Lu S, Bao MY, Miao SM, Zhang X, Jia QQ, Jing SQ, Shan T, Wu XH, Liu Y (2019) Prevalence of hypertension, diabetes, and dyslipidemia, and their additive effects on myocardial infarction and stroke: a cross-sectional study in Nanjing. China Ann Transl Med 7:436. https://doi.org/10.21037/atm.2019.09.04

Muhammad S, Aller MI, Maser-Gluth C, Schwaninger M, Wisden W (2010) Expression of the kcnk3 potassium channel gene lessens the injury from cerebral ischemia, most likely by a general influence on blood pressure. Neuroscience 167:758-764. https://doi. org/10.1016/j.neuroscience.2010.02.024

$\mathrm{Pj}$ T, KJ B (2013) Oxygen and mitochondrial inhibitors modulate both monomeric and heteromeric TASK-1 and TASK-3 channels in mouse carotid body type-1 cells. J Physiol 591:5977-5998

Samal BB, Waites CK, Almeida-Suhett C, Li Z, Marini AM, Samal NR, Elkahloun A, Braga MF, Eiden LE (2015) Acute response of the hippocampal transcriptome following mild traumatic brain injury after controlled cortical impact in the rat. J Mol Neurosci 57:282-303. https://doi.org/10.1007/s12031-015-0626-2

Shaper NL, Lin SP, Joziasse DH, Kim DY, Yang-Feng TL (1992) Assignment of two human alpha-1,3-galactosyltransferase gene sequences (GGTA1 and GGTA1P) to chromosomes 9q33-q34 and 12q14-q15. Genomics 12:613-615. https://doi.org/10.1016/ 0888-7543(92)90458-5

Smyth GK (2005) Limma: linear models for microarray data. Bioinformatics and computational biology solutions using $\mathrm{R}$ and Bioconductor. Springer, Berlin, pp 397-420

Sun MS, Jin H, Sun X, Huang S, Zhang FL, Guo ZN, Yang Y (2018) Free radical damage in ischemia-reperfusion injury: an obstacle in acute ischemic stroke after revascularization therapy. Oxid Med Cell Longev 2018:3804979. https://doi.org/10.1155/2018/38049 79

Suzuki M, Takaishi S, Nagasaki M, Onozawa Y, Iino I, Maeda H, Komai T, Oda T (2013) Medium-chain fatty acid-sensing receptor, GPR84, is a proinflammatory receptor. J Biol Chem 288:1068410691. https://doi.org/10.1074/jbc.M112.420042

Venkat P, Chopp M, Chen J (2017) Blood-brain barrier disruption, vascular impairment, and ischemia/reperfusion damage in diabetic stroke. J Am Heart Assoc. https://doi.org/10.1161/jaha.117. 005819

Venkataraman C, Kuo F (2005) The G-protein coupled receptor, GPR84 regulates IL-4 production by T lymphocytes in response to $\mathrm{CD} 3$ crosslinking. Immunol Lett 101:144

Xiong X, Barreto GE, Xu L, Ouyang YB, Xie X, Giffard RG (2011) Increased brain injury and worsened neurological outcome in interleukin-4 knockout mice after transient focal cerebral ischemia. Stroke 42:2026-2032. https://doi.org/10.1161/strok eaha. 110.593772

Yang IS, Kim S (2015) Analysis of whole transcriptome sequencing data: workflow and software. Genom Inform 13:119-125. https:// doi.org/10.5808/gi.2015.13.4.119

Publisher's Note Springer Nature remains neutral with regard to jurisdictional claims in published maps and institutional affiliations. 